\title{
The potential of photoacoustic techniques in inflammatory arthritis: what can it do to assist conventional imaging methods?
}

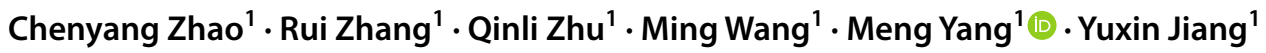

Received: 29 March 2020 / Revised: 18 February 2021 / Accepted: 4 April 2021 / Published online: 6 May 2021

(c) The Author(s) 2021

\begin{abstract}
To make comprehensive assessments of some rheumatoid diseases, a more reliable imaging method for evaluating joint lesions is required. Photoacoustic imaging (PAI) is a state-of-the-art imaging technique, providing new options for diagnosing joint disease. In light of the recent preclinical studies, detailed morphological structures and micro-vessels of small joints, especially the finger joints, could be visualized by PAI with high spatial resolution and optical contrast using different PA implementations. By measuring the signals of oxygenated and deoxygenated hemoglobin through dual-wavelength PAI, oxygenation of local tissues can be identified, thus enabling functional PA imaging. Notably, among various PA systems, the co-registration of PAI into high-end commercial US units with a special-designed handheld PA/US probe can be of great value in further clinical application. Molecular PAI of small joints can also be performed using exogenous PA chromophores. In this article, we aim to inform readers of this new technique in joint imaging and its potential use, to impart a deeper understanding of medical imaging in this field.
\end{abstract}

Keywords Diagnostic imaging $\cdot$ Photoacoustic techniques $\cdot$ Ultrasonography $\cdot$ Joint imaging $\cdot$ Arthritis

\begin{tabular}{ll}
\multicolumn{2}{l}{ Abbreviations } \\
PAI & Photoacoustic imaging \\
PACT & Photoacoustic computed tomography \\
OA & Osteoarthritis \\
RA & Rheumatoid arthritis \\
CT & Computed tomography \\
MRI & Magnetic resonance imaging \\
US & Ultrasound \\
CEUS & Contrast-enhanced ultrasound \\
DOI & Diffuse optical imaging \\
LED & Light-emitting diode \\
PRF & Pulse repetition rate \\
OPO & Optical parametric oscillator \\
PAM & Focused-scanning photoacoustic microscopy \\
PAE & Photoacoustic endoscopy \\
DIP joint & Distal interphalangeal joint \\
MCP joint & Metacarpophalangeal joint \\
SNR & Signal-noise ratio
\end{tabular}

Meng Yang

yangmeng_pumch@126.com

1 Department of Ultrasound, Peking Union Medical College Hospital, Peking Union Medical College and Chinese Academy of Medical Sciences, Shuaifuyuan No.1, Dongcheng District, Beijing 100730, China

\section{Arthritis and current joint imaging techniques}

This decade has witnessed a growing prevalence of arthritis all around the world, including rheumatoid arthritis (RA) and osteoarthritis (OA), imposing a considerable burden on medical care. The incidence rate of RA in China is approximately $0.42 \%$, presenting an accelerating trend compared with previous years [1]. The estimated prevalence of symptomatic knee $\mathrm{OA}$ is $8.1 \%$, which also posed a serious health problem [2]. Early diagnosis and accurate assessments of disease activity of arthritis can be essential for timely medical intervention to prevent the deterioration of arthritis and aggressive disabilities, which can be difficult for current laboratory and imaging methods [3-6]. The development of noninvasive imaging techniques, capable of helping in the early diagnosis and evaluation of arthritis, has triggered increasing interest among researchers.

In current clinical practice, magnetic resonance imaging (MRI) and high-frequency ultrasound (US), which have good performance in visualizing soft tissues, are the common imaging methods for assistant diagnosis and evaluation of joint disease, based on the pathological changes within the inflamed tissues, such as joint space narrowing, subchondral sclerosis, marginal osteophytes of OA, synovial 
thickening, hyperemia, and bone erosions of RA [7, 8]. Nevertheless, the role of conventional US in arthritis has not been established yet, due to conflicting research results and shortage of large-scale clinical studies, and the utilization of Doppler US is still unable to detect early synovitis with the formation of micro-vessels [9]. Magnetic resonance imaging (MRI) may help identify bone damage and synovitis in the early stage of RA, but its clinical utilization of MRI is limited by its high cost and inconvenience [10,11]. Therefore, new imaging techniques for joint imaging to visualize articular inflammation are in need of improving the clinical management of RA and OA.

Recently, photoacoustic imaging (PAI), a new noninvasive and nonionizing optical imaging method, capable of implementing morphological, functional and molecular imaging, has emerged. Due to the versatile imaging capabilities of PAI, researchers have shown an increased interest in the clinical utilization of PAI, including the field of joint imaging. To illustrate the recent advancement of PAI in joint imaging and its potential use in evaluating arthritis, we searched the literature from 2006 to 2020 in PubMed and EMBASE and made a brief summary of these new investigations. Also, some of the results from our research team of Peking Union Medical College Hospital were also added in this article. We hope that this review will contribute new insight into the applications of PAI in joint imaging.

\section{Photoacoustic imaging (PAI)}

PAI, a hybrid imaging technique, combines optical imaging systems and ultrasound modalities by emitting lasers in an adjustable range of electromagnetic wavelengths and receiving ultrasonic signals for image formation [12]. The establishment of PA techniques has led to a proliferation of studies about this new imaging method.

The photoacoustic effect, the theoretical basis of PA imaging, was demonstrated in the late 19th century by Alexander Graham Bell [13]. Briefly, the PA effect is the transition of light into sound information. When tissues are irradiated by short-pulsed laser light, the inner temperature of the tissue will rise after the absorption of the light energy, followed by a thermo-elastic expansion. A wide-band of ultrasound waves is induced by the localized pressure changes and captured by a single-element ultrasonic transducer or transducer arrays. The signals are then translated into PA images through an image reconstruction algorithm [14] (Fig. 1). Different biological tissues can be visualized by PAI through their distinct optical absorption contrasts. Unlike conventional optical imaging, PAI has a high spatial resolution with a superior penetration depth due to the weak scattering of ultrasound waves. Therefore, morphological and functional imaging based on the optical absorption of different components can be archived by PAI, with high optical contrast and microscale resolution at a fairly good penetration depth, making it a unique medical imaging method with potential clinical values $[15,16]$. And in the last decade, the use of PAI has rapidly evolved from laboratory to a variety of clinical settings $[17,18]$.

A typical PAI system mainly consists of an optical system for laser emission and regulation, and a processing system for receiving and analyzing ultrasonic signals [19]. An optical parametric oscillator (OPO) pumped by Q-switched neodymium-doped yttrium aluminum garnet (Nd:YAG) laser, dye laser systems and) can be utilized as an ideal light excitation source for PAI in biological tissues, as well as a commercially available light-emitting diode (LED) [20, 21]. Pulsed lasers diode (PLD), a type of lighter and cheaper excitation resources has also been explored for PAI recently [22]. The light delivery system is made up of a series of lenses and optical fibers, arranged in different geometries [23]. Ultrasonic transducers, such as piezoelectric transducers ultrasonic transducers, and capacitive micromachined ultrasonic transducers are employed for receiving the ultrasonic signals generated from PA effect [24].

Intrinsic chromophores inside the human body can be observed by PAI at multiple wavelengths, including oxygenated and deoxygenated hemoglobin, melanin, water, lipid,
Fig. 1 Schematic of PA principle (Created with BioRender. com)

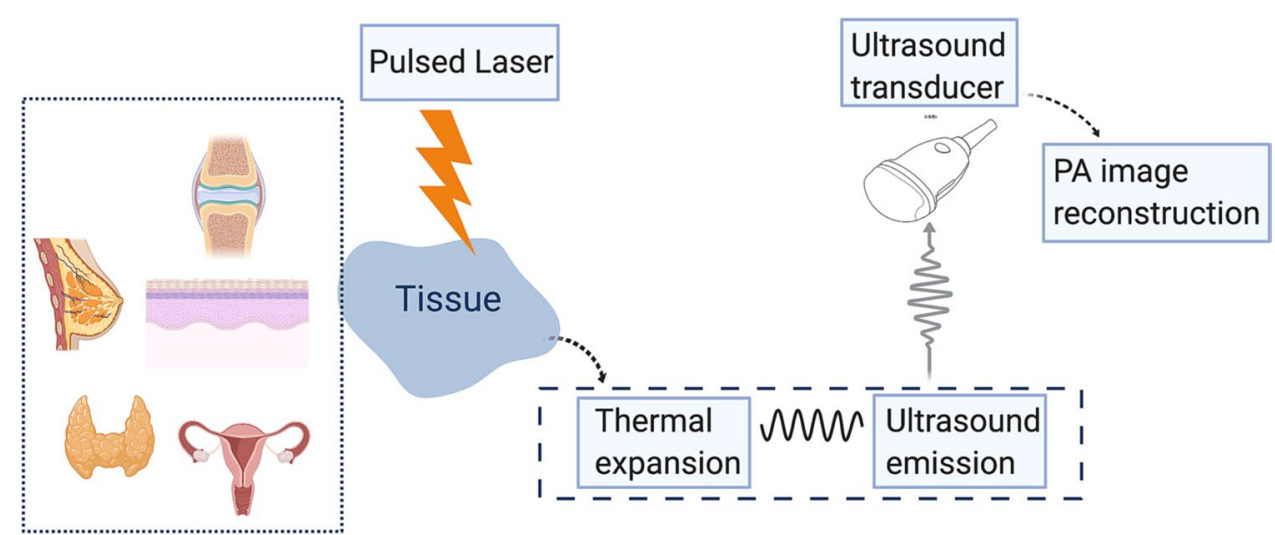


and other substances [25]. Using quantifying oxygenated and deoxygenated hemoglobin in the visible spectral range (450-600 nm), some functional indexes of the regional tissues can be measured, which achieves morphological and functional imaging simultaneously $[15,16]$. There are also emerging in vivo experiments about the utilization of exogenous optical contrast agents in PAI, including the biomaterial dyes, such as indocyanine green (ICG), gold nanoparticles, single-walled carbon nanotubes (SWNTs) [26, 27]. Molecular PAI can be realized through encapsulating the exogenous agents with targeted particles, facilitating the early diagnosis of some diseases, and the monitoring of drug delivery monitoring $[28,29]$. And molecular PAI is experiencing a fast advancement along with the development of nanotechnology [30].

The major types of PAI implementations fit in photoacoustic computed tomography (PACT), focused-scanning photoacoustic microscopy (PAM), and photoacoustic endoscopy (PAE) [31]. The potential clinical utilities of PACT have been widely explored by many animal experiments and preclinical studies [32, 33]. PAI can be combined with other imaging techniques to establish powerful hybrid multifunctional imaging modalities, including US, DOI, and MRI. Among these hybrid modalities, the integration of PA and US has been reported to show remarkable potential in clinical promotion, such as breast cancer, thyroid tumors, and mapping sentinel lymph nodes [23, 34-36].

\section{The explorations of PAI in inflammatory arthritis}

These advantages of PAI, illustrated above make it an eligible method for detecting inflammatory arthritis. In the last decade, there has been an increasing interest in the utilization of PAI in joint imaging and several studies were carried out. Several PA implementations for joint imaging have been developed, and the feasibility was validated by a series of preclinical and clinical experiments (Table 1). This review will give a glimpse of the PAI techniques mainly applied in small joints, providing an outlook of the future clinical translation of PAI in this field.

\section{Conventional photoacoustic computed tomography (PACT) for joint imaging}

\section{PACT systems for single PA imaging}

Reconstruction-based PACT is one of the main categories of PA systems. Current PACT prototypes in joint imaging can provide $3 \mathrm{D}$ reconstruction through transducer rotation, and semispherical or spherical transducer-arrays. Xueding Wang et al. introduced a photoacoustic tomography modality for joint imaging in 2006 [37]. The imaging system was composed of an OPO laser generation, pulsed by an Nd:YAG laser pulse, and a wideband ultrasonic transducer with a central frequency of $10 \mathrm{MHz}$. In this system, a full circular scan on rat tails through the rotation of the ultrasound transducer was performed for $2 \mathrm{D}$ and $3 \mathrm{D}$ reconstruction of peri-articular tissues. The distribution of hemoglobin in the local tissues was visualized and quantified by the PAI at the

Table 1 Researches for PAI in joint imaging and inflammatory arthritis

\begin{tabular}{lllc}
\hline Author & Year & Modality & Models \\
\hline Xueding Wang [37, 38] & 2006,2008 & 3D PACT & AIA rats \\
Yao Sun [40, 43] & 2009,2011 & 3D PACT & $\begin{array}{l}\text { Phantoms } \\
\text { Human fingers }\end{array}$ \\
Sergey Ermilov [45] & 2012 & & Human fingers \\
Peter Van Es [44] & 2014 & 3D PACT & Human fingers \\
Huang [68] & 2012 & 3D PACT & Human fingers \\
Yubin Liu [47] & 2016 & 3D PACT & Human fingers \\
Milan Oeri [48] & 2017 & PACT/USCT & Human fingers \\
Heng Guo [49] & 2019 & PACT/USCT & AIA rats and human fingers \\
Xueding Wang [50, 53], Jo J [56] & $2011-2013,2017$ & PACT/USCT & AIA rats, human fingers of \\
& & Dual-modal PA/US system & healthy individuals and \\
arthritis patients & Human fingers of healthy \\
individuals and arthritis & patients
\end{tabular}


wavelength of $700 \mathrm{~nm}$ where hemoglobin had the strongest absorption rate [38]. The feasibility of this PACT system in imaging human finger joints was also validated. The wavelength was set at $720 \mathrm{~nm}$ to acquire good visualization of the distal interphalangeal (DIP) and proximal interphalangeal (PIP) joint, and the maximum spatial resolution was $52 \mu \mathrm{m}$ in the in-plane 2D imaging [39].

A quantitative 3D PACT device with dedicated designed rotation steps of the rotatory arms, produced by Sun Y et al., completed the $3 \mathrm{D}$ reconstruction of PA images on a joint phantom and the distal interphalangeal (DIP) joint of a healthy volunteer [40-42]. Intricate structures of joint tissues and interarticular spaces could be visualized and calibrated. The PACT system was applied in vivo detection of OA in the human DIP finger joint, with a spatial resolution of approximately $0.5 \mathrm{~mm}$ [43]. The thickness of the joint cavity of the OA patients were calibrated on 3D PA imaging with a mean value of $1.3 \mathrm{~mm}$, less than that of healthy joints $(2.0 \mathrm{~mm})$.

Not only were the detailed structures of small joints visualized by the PACT systems, the perfusion of micro-vessels and angiogenesis, the hallmarks of inflammatory arthritis, could also be distinguished on PA images. A PACT modality with an in-plane resolution of $100 \mu \mathrm{m}$ designed by Peter Van Es et al. successfully visualized small vascular structures of finger joints, ranging from the diameter of $1.5 \mathrm{~mm}$ to threadlike vessels beneath the dermal layer in cross-sectional PA slices [44]. Sergey Ermilov also developed a new PACT system for delineating microangiography to the capillary level of the finger joints in 3D PA imaging at the wavelength of $700-800 \mathrm{~nm}$ [45]. The system might be also useful for monitoring the vasomotor activity of finger joints by visualizng the thermoregulatory response of small vessels through a quick serial PA scan.

\section{PACT systems integrating PA and US imaging}

Both PA signals for PA imaging and ultrasonic signals for US imaging can be received and processed by ultrasonic transducers. Therefore, simultaneous PA and US imaging can be recovered by integrating the PA technique into ultrasound imaging systems. The dual-modality PA/US systems, a combination of PA imaging and US imaging, has intrigued enormous interest from researchers recently. Yoshihiro Hagiwara et al. developed a dual PA/US system that could perform a mechanical scanning of the immobilized knee joints of rats through the movement of an ultrasound transducer at the wavelength of $532 \mathrm{~nm}$. Increasing PA signal intensity of the subchondral bone and articular cartilage was visualized, whereas the US signals were weak [46].

Yubin Liu et al. developed another dual-modal PA/ US imaging, which successfully displayed the anatomical structures of the PIP joint of a healthy volunteer at the wavelengths of $680 \mathrm{~nm}, 720 \mathrm{~nm}, 760 \mathrm{~nm}$, and $800 \mathrm{~nm}$, and the soft tissues and small blood vessels were clearly visualized at all wavelengths. The hard tissues, such as the phalanx, could be observed at $680 \mathrm{~nm}$ [47]. Based on the same principles, Milan Oer et al. and Heng Guo et al. also designed a dual-modality PA/US system, that could make assessment of the micro-vessels within the finger joints [48, 49].

\section{The integration of PA techniques into commercialized ultrasonic systems}

Most of the current photoacoustic systems, introduced in recent years, were often comprised of large and costly apparatus that restrain their future use in clinical conditions. Researchers are determined in developing new types of dual-modality PA/US systems and integrating PA techniques into commercialized ultrasound systems for further clinical application of PAI. This kind of dual-modal imaging system permits noninvasive real-time PA and US imaging simultaneously based on high-end US device. It takes advantage of the compactness and high cost-efficiency of commercial ultrasound systems and shows great promise in future clinical promotion of PA techniques. The feasibility of the dual-modality PA/US system in the evaluation of joint disease has been explored by recent studies [50-53].

A stand-alone US unit was first applied to the co-registration with PA devices. A linear probe driven by a commercial US system was modified to receive PA signals in a special geometry [50]. Significantly increased PA signals were detected in the ROI of arthritis ankles compared with the healthy ankles at wavelengths of 1064 and $532 \mathrm{~nm}$ [51]. A prominent decrease in PA signal intensities was observed in the arthritic rats after drug treatment, indicating the potential of the dual-modality system in treatment monitoring [52]. Structural imaging of the human finger joint was also successful on the dual-modality PA/US system, after several modifications for better adaptation in clinical use [53].

The design of PA/US probe, the integration of a PA illuminating system into a handheld ultrasonic probe, can be a decisive step for the improvement of the dual-mode PA/ US imaging system, which facilitates noninvasive real-time PA and US imaging in a more easy and manageable way for clinicians. K. Daoudi and P.J. van den Berg presented a compact dual-modality PA/US system, made up of a sophisticated commercial ultrasonic system with a handheld PA probe [54]. Laser beams were dedicatedly reshaped to a rectangular form to adjust the shape of the probe. Gray-scale PA images and pseudo-color PA signals superimposed on US images were presented, showing the small blood vessels and bone surfaces of small joints with a satisfactory resolution. In a clinical study of this system on the PIP joints of 10 RA patients, the PA signals from the hypertrophic joint 
spaces were quantitatively assessed, which appeared to be well-correlated with PDUS scoring [55].

Xueding Wang et al. developed another state-of-the-art hybrid imaging modality in 2016 that performs synchronous PA and US imaging on a commercial US unit using a handheld linear probe. It was preliminarily validated on human finger joints at multiple wavelengths, showing the PA images of osseous structures, tendons, and cartilages. The blood vessels around the surface of these structures were also delineated in PA mode, providing additional functional information by evaluating blood oxygen saturation [53]. Later in 2017, the group utilized the system in assessing inflammatory arthritis of the RA patients [56]. The content of hemoglobin within the surrounding soft tissues of the lesioned metacarpophalangeal (MCP) joints was measured by the system at a wavelength of $580 \mathrm{~nm}$. Tissue oxygenation was calculated by quantifying the PA signals of oxygenated-hemoglobin and deoxygenated-hemoglobin at the wavelengths of $532 \mathrm{~nm}$ and $1064 \mathrm{~nm}$ respectively. The research team also developed a LED-based PA/US system with a higher signal-noise ratio (SNR) which was able to detect the micro-vessels up to $5 \mathrm{~mm}$ within the finger. The PA signals at wavelengths of 690 and $850 \mathrm{~nm}$ were measured for quantifying the blood oxygenation of the inflamed tissues. As a cost-effective excitation resource, the use of LED in PA/US systems may further facilitate the clinical adoption of PAI [57].

Our team, from the Department of Ultrasound, Peking Union Medical College Hospital, and Shenzhen Mindray Bio-Medical Electronics Co., Ltd., is also focusing on the development of a hybrid PA and US imaging platform, on a high-end commercial US unit. Different from the current dual-modal PA/US modalities, which has relatively lowquality images, and only combines PAI with B-mode US, the system is designed to co-register PAI and multiple US modes, including color-Doppler, power-Doppler ultrasound, and other ultrasonic techniques (Fig. 2). This high-end imaging platform has been successfully tested on the imaging of thyroid nodules and breast cancer [58]. In a preliminary clinical study, we performed multi-modal PA/US imaging on 31 RA patients, and PA parameters, including semi-quantitative PA scores and oxygen saturation $\left(\mathrm{SO}_{2}\right)$, were found to present good correlations with standard clinical scores [59] (Fig. 3).

\section{Hybrid imaging systems combining PACT and other special imaging methods}

Studies have also attempted to integrate the PA technique with other optical imaging techniques. A hybrid system integrating PA and diffuse optical tomography (DOT) with high-powered reconstruction algorithms was designed and performed on human finger joints by $\mathrm{Xi} \mathrm{L}$ et al. The

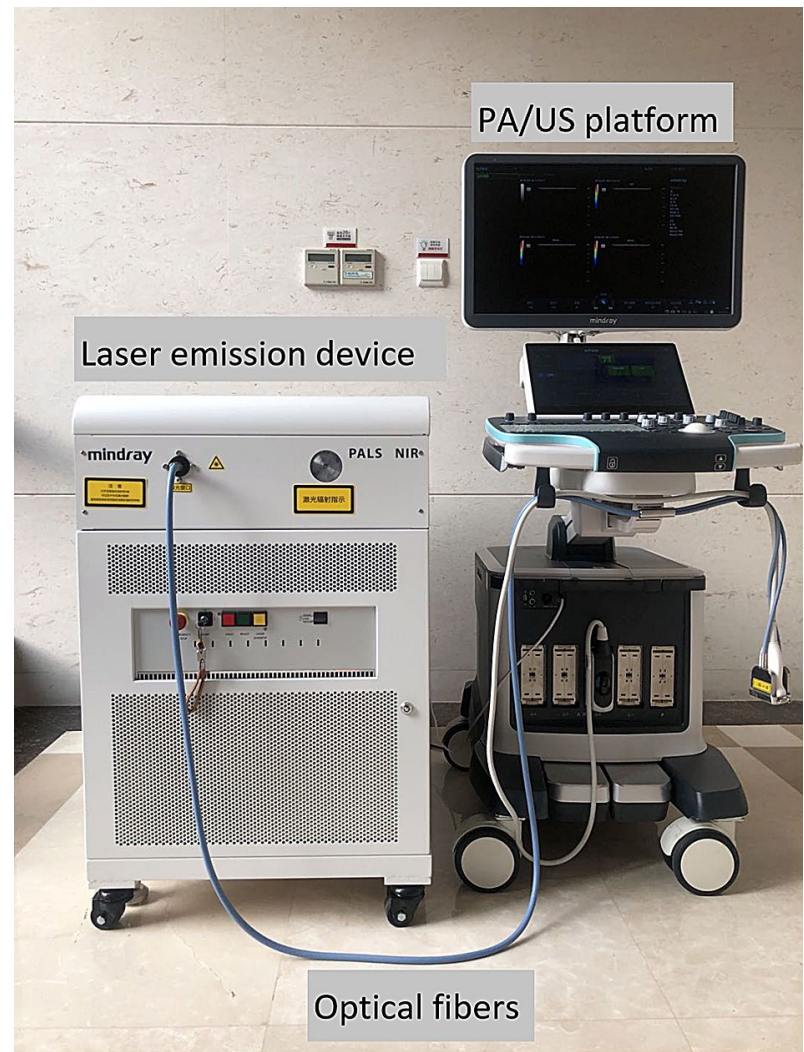

Fig. 2 Schematic of the multi-modal PA/US system [59]

structures of the phalanx and surrounding soft tissues were reconstructed by PACT, and optical contrast and reduced scattering co-efficiencies of DOT were obtained [60].

\section{Molecular PA imaging for inflammatory arthritis}

Exogenous contrast agents can facilitate molecular PAI in arthritis by binding specific molecules to those PA dyes, enabling visualizing inflammatory changes of local joints at the molecular level, as well as delivering therapeutic agents for arthritis.

According to Ray et al. [61], Seminaphthorhodafluor (SNARF-5F), a type of pH-sensitive dye, was amalgamated with nanoprobes and quantified by detecting the PA signals from the nanoprobes to calculate the $\mathrm{pH}$ levels in the joint tissues of a rat tail. The $\mathrm{pH}$-sensitive nanoprobes could also be developed to be tissue- or cell-specific. An L-selectin/Pselectin targeting compound combining an exogenous PA contrast was designed to detect inflammation on PAI by Beziere $\mathrm{N}$. The distribution of PA signals in the knee and ankle joints of arthritic mice was in accordance with MRI and pathological results [62]. Chamberland DL et al. conjugated gold nanorods, a common exogenous optical contrast, with molecules of etanercept for the purpose of monitoring drug delivery [63]. The compounds were injected in the 


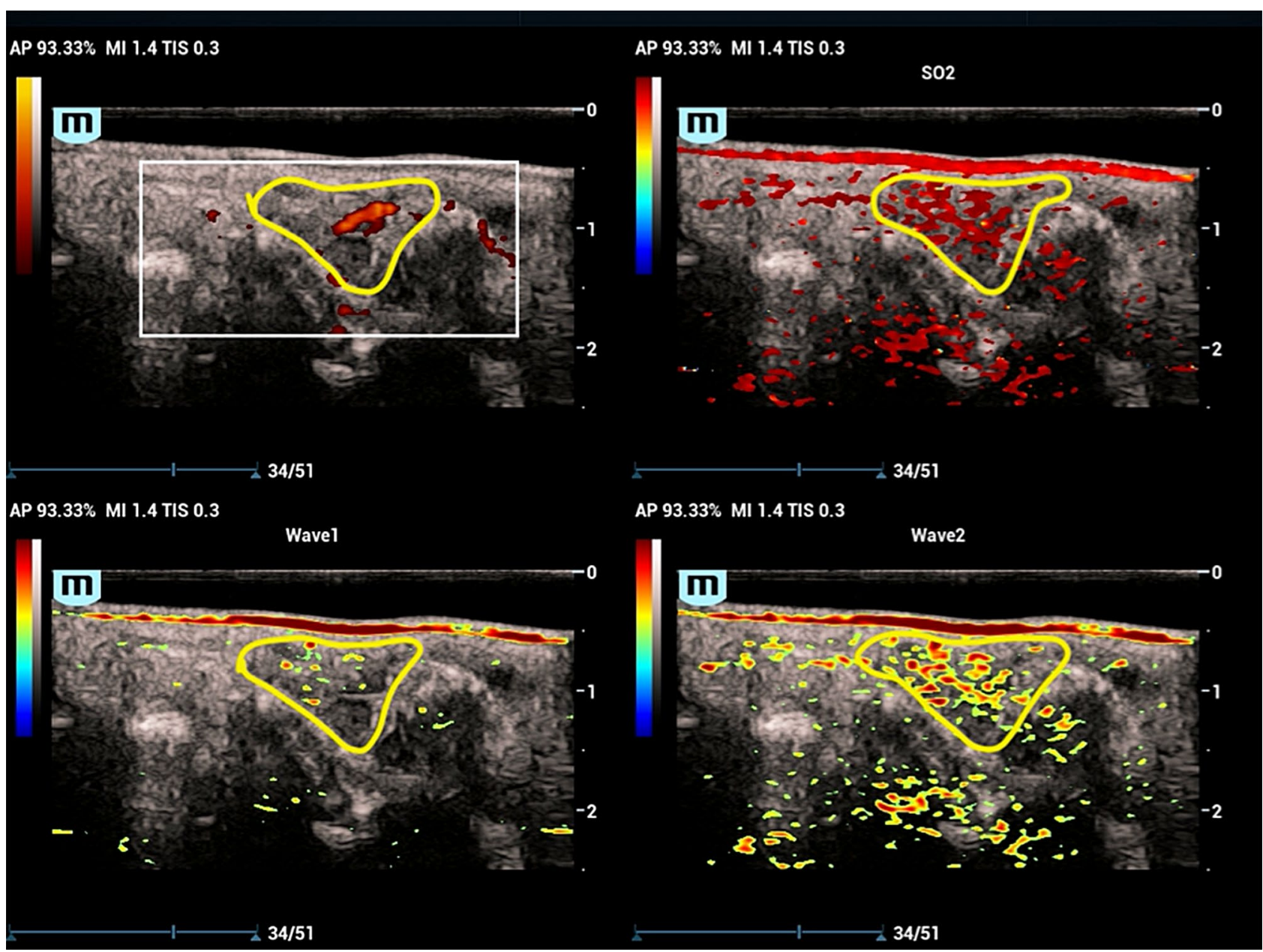

Fig. 3 The PA/US images of the dorsal aspect of the wrist of a RA patient. In the PA mode, abundant optical signals were visualized at both the wavelengths of $750 \mathrm{~nm}$ and $830 \mathrm{~nm}$ (c, d), representing hyperemia of the inflamed lesion (the region marked with the yellow

rat tail joints to receive PA examination, in an absorption peak of $600 \mathrm{~nm}$, at which hemoglobin has a low absorption rate. After the intra-articular injection, the profiles of the connective tissues surrounding the tail joints were contoured on PAI. The potential of PAI in the monitoring of drug delivery and therapeutic efficacy within the local soft tissues of joints could be expected. Notably, without combining imaging molecules, Clofazimine (CFZ), an FDAapproved anti-inflammatory drug, was found to be able to be monitored by PAI directly at the wavelength of $450 \mathrm{~nm}$ [64]. To integrating molecular PA imaging and dual-modal PA/US imaging, our team fabricated VEGF-targeted PA/US microbubbles, by loading the ultrasonic microbubbles with Cy5.5-decorated VEGF-antibodies in an in-vivo experiment. CEUS and PAI were performed on the AIA rats by injecting the multi-functional microbubbles, and the high expressions of VEGF within local tissues on the inflamed paws of AIA rats were validated and quantitatively evaluated [65] (Fig. 4). line). More signals were presented in PA mode than power Doppler US (a). In the SO2 part, the signals were demonstrated in the pseudocolor of red (b), with a relative $\mathrm{SO} 2$ value of $97.33 \%$, classified as hyperoxia [59]

\section{The clinical prospect of PAI for joint imaging}

As a newly presented imaging technique, PAI is still being developed in a preliminary stage. Several clinical studies have been launched to validate the feasibility of these PAI systems in assessing arthritis. Yao Sun et al. performed the first clinical assessment of the 3D PACT system in the quantitative evaluation of joint spaces of the DIP joints in OA patients [43]. Pim J. van den Berg et al. evaluated the clinical potential of the portable PA/US system on 10 RA patients with evident synovitis. PA signals were successfully quantified, presenting a strong agreement with power-Doppler US [55]. Janggun Jo et al. performed both morphological and functional imaging of inflamed finger joints in RA patients by quantifying hemoglobin and oxygenation at multiple wavelengths [56]. Our team also performed multi-modal PA/US imaging on 31 RA patients using a multi-modal PA/US imaging system with a handheld PA/US probe, and the semi-quantitative PA 


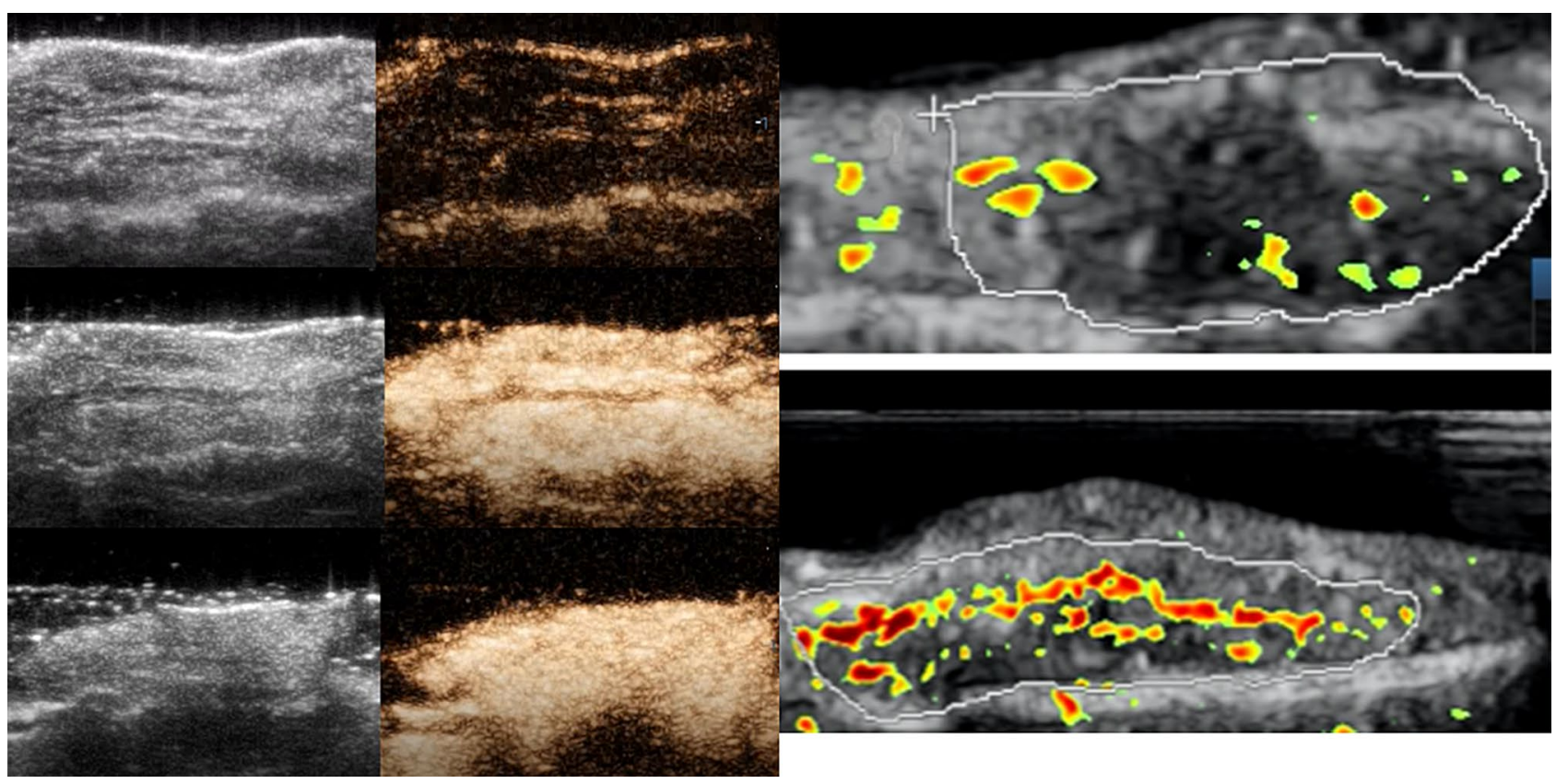

Fig. 4 The Contrast-enhanced ultrasound (CEUS) and PA images of the inflamed paws of AIA rats before and after the injection of VEGF-targeted PA/US microbubbles. a The CEUS imaging after the injection of the microbubbles at 0,10 and $70 \mathrm{~s}$, remarkable enhance- ment is presented; $\mathbf{b}$ PA imaging before and after injection of targeted MBs; Significant increasement of PA signals was observed within the inflamed region (heighted with white lines) after injection [65] scores and oxygen saturation $\left(\mathrm{SO}_{2}\right)$ were found to be correlated with standard clinical scores. Although the investigations of PAI are currently limited, this promising tool has leveraged the platform of joint imaging and provides us with a new option for functional and molecular imaging for joint disease, especially arthritis. Among these prototypes of PA techniques for joint imaging, the co-registration of PA and US systems with a portable probe, by virtue of its utilization of the stability and advancement of commercial US units, can be an essential step for future clinical translation and promotion. Further adjustments are required for improving the imaging quality of PAI, which may not perform as well as some highly-established imaging methods, such as MRI [66]. Researchers are optimizing the elements of PA systems to achieve higher spatial resolution and SNR. Some studies have begun working on eliminating the artifacts from the US absorptions of bone surfaces [67]. Additionally, a standard protocol for performing PAI and quantitative analysis of 3D PA imaging is also crucial for clinical application.

\section{Conclusion}

PAI, a novel multi-functional imaging technique, has generated great interest form researchers in the field of medical imaging. The penetration depth, optical contrast and spatial resolution of PAI are all-sufficient for visualizing the morphological structures of peripheral joints, especially for small joints. By measuring the content of oxygenated-hemoglobin and deoxygenated-hemoglobin at two wavelengths, blood oxygenation can be calculated, allowing functional evaluation of the joint tissues. The exogenous PA contrast agent, targeted with some specific molecular biomarkers, makes it possible to conduct molecular imaging with the use of PA modalities. This review presented different types of newly developed PA systems for joint imaging, including PACT, dual-modality PA/US systems, and a combination of PA techniques with other imaging methods. The phantom studies, animal studies and clinical studies of PAI indicated its clinical potential. We also discussed some special applications of PAI in molecular imaging of joint diseases in this article. These developments in PAI have heightened the need for further clinical validation of this method.

Acknowledgements This work was funded by Beijing Natural Science Foundation (JQ18023); National Natural Science Foundation of China (81421004, 81301268); Beijing Nova Program Interdisciplinary Cooperation Project (xxjc201812); International S\&T Cooperation Program of China (2015DFA30440); Beijing Nova Program (Z131107000413063).

Funding This work was funded by Beijing Natural Science Foundation (JQ18023); National Natural Science Foundation of China (81421004, 81301268); Beijing Nova Program Interdisciplinary Cooperation 
Project (xxjc201812); International S\&T Cooperation Program of China (2015DFA30440); Beijing Nova Program (Z131107000413063).

Availability of data and material The data and material used in the study are available from the corresponding author on reasonable request.

\section{Declarations}

Conflict of interest The authors declare that they have no conflict of interest.

\section{Ethical approval NA}

Consent for publication All of the authors have agreed with the publication.

Open Access This article is licensed under a Creative Commons Attribution 4.0 International License, which permits use, sharing, adaptation, distribution and reproduction in any medium or format, as long as you give appropriate credit to the original author(s) and the source, provide a link to the Creative Commons licence, and indicate if changes were made. The images or other third party material in this article are included in the article's Creative Commons licence, unless indicated otherwise in a credit line to the material. If material is not included in the article's Creative Commons licence and your intended use is not permitted by statutory regulation or exceeds the permitted use, you will need to obtain permission directly from the copyright holder. To view a copy of this licence, visit http://creativecommons.org/licenses/by/4.0/.

\section{References}

1. Xiaofeng ZE, Aichun TA, Xiaoping XI. Disease burden and quality of life of rheumatoid arthritis in China: a systematic review. Chin J Evid-Based Med. 2013;13(3):300-7.

2. Tang X, Wang S, Zhan S, Niu J, Tao K, Zhang Y, et al. The prevalence of symptomatic knee osteoarthritis in China: results from the China Health and Retirement Longitudinal Study. Arthritis Rheumatol. 2016;68(3):648-53.

3. Cross M, Smith E, Hoy D, Nolte S, Ackerman I, Fransen M, et al. The global burden of hip and knee osteoarthritis: estimates from the global burden of disease 2010 study. Ann Rheum Dis. 2014;73(7):1323-30.

4. Prieto-Alhambra D, Judge A, Javaid MK, Cooper C, DiezPerez A, Arden NK. Incidence and risk factors for clinically diagnosed knee, hip and hand osteoarthritis: influences of age, gender and osteoarthritis affecting other joints. Ann Rheum Dis. 2014;73(9):1659-64.

5. Minichiello E, Semerano L, Boissier MC. Time trends in the incidence, prevalence, and severity of rheumatoid arthritis: a systematic literature review. Joint Bone Spine. 2016;83(6):625-30.

6. Rheumatoid arthritis. Nat Rev Dis Primers. 2018;4:18002. https:// doi.org/10.1038/nrdp.2018.2.

7. Czyrny Z. Standards for musculoskeletal ultrasound. J Ultrasonogr. 2017;17(70):182-7.

8. Hayashi D, Roemer FW, Guermazi A. Imaging of osteoarthritis: recent research developments and future perspective. Br J Radiol. 2017. https://doi.org/10.1259/bjr.20170349.

9. Caporali R, Smolen JS. Back to the future: forget ultrasound and focus on clinical assessment in rheumatoid arthritis management. Ann Rheum Dis. 2018;77(1):18-20.

10. Ostergaard M, Bird P, Gandjbakhch F, Eshed I, Haugen IK, Haavardsholm EA, et al. The OMERACT MRI in Arthritis
Working Group: update on status and future research priorities. J Rheumatol. 2015;42(12):2470-2.

11. Nieuwenhuis WP, van Steenbergen HW, Mangnus L, Newsum EC, Bloem JL, Huizinga TWJ, et al. Evaluation of the diagnostic accuracy of hand and foot MRI for early Rheumatoid Arthritis. Rheumatology (Oxford). 2017;56(8):1367-77.

12. Yao J, Wang LV. Photoacoustic tomography: fundamentals, advances and prospects. Contrast Media Mol Imaging. 2011;6(5):332-45.

13. Beauvoit B, Evans SM, Jenkins TW, Miller EE, Chance B. Correlation between the light scattering and the mitochondrial content of normal tissues and transplantable rodent tumors. Anal Biochem. 1995;226(1):167-74.

14. Zhou Y, Yao J, Wang LV. Tutorial on photoacoustic tomography. J Biomed Opt. 2016;21(6):61007.

15. Wang X, Pang Y, Ku G, Xie X, Stoica G, Wang LV. Noninvasive laser-induced photoacoustic tomography for structural and functional in vivo imaging of the brain. Nat Biotechnol. 2003;21(7):803-6.

16. Zackrisson S, van de Ven S, Gambhir SS. Light in and sound out: emerging translational strategies for photoacoustic imaging. Can Res. 2014;74(4):979-1004.

17. Zhao T, Desjardins AE, Ourselin S, Vercauteren T, Xia W. Minimally invasive photoacoustic imaging: current status and future perspectives. Photoacoustics. 2019;16:100146.

18. Steinberg I, Huland DM, Vermesh O, Frostig HE, Tummers WS, Gambhir SS. Photoacoustic clinical imaging. Photoacoustics. 2019;14:77-98.

19. Wang $\mathrm{LV}, \mathrm{Hu} \mathrm{S}$. Photoacoustic tomography: in vivo imaging from organelles to organs. Science (New York, NY). 2012;335(6075):1458-62.

20. Maslov K, Wang LV. Photoacoustic imaging of biological tissue with intensity-modulated continuous-wave laser. J Biomed Opt. 2008;13(2):024006.

21. Allen TJ, Beard PC. High power visible light emitting diodes as pulsed excitation sources for biomedical photoacoustics. Biomed Opt Express. 2016;7(4):1260-70.

22. Upputuri PK, Pramanik M. Fast photoacoustic imaging systems using pulsed laser diodes: a review. Biomed Eng Lett. 2018;8(2):167-81.

23. Kim C, Erpelding TN, Jankovic L, Pashley MD, Wang LV. Deeply penetrating in vivo photoacoustic imaging using a clinical ultrasound array system. Biomed Opt Express. 2010;1(1):278-84.

24. Valluru KS, Willmann JK. Clinical photoacoustic imaging of cancer. Ultrasonography (Seoul, Korea). 2016;35(4):267-80.

25. Guo Z, Hu S, Wang LV. Calibration-free absolute quantification of optical absorption coefficients using acoustic spectra in 3D photoacoustic microscopy of biological tissue. Opt Lett. 2010;35(12):2067-9.

26. Yao J, Wang LV. Recent progress in photoacoustic molecular imaging. Curr Opin Chem Biol. 2018;45:104-12.

27. Fu Q, Zhu R, Song J, Yang H, Chen X. Photoacoustic imaging: contrast agents and their biomedical applications. Adv Mater. 2019;31(6):e1805875.

28. Zhang Y, Yu J, Kahkoska AR, Gu Z. Photoacoustic drug delivery. Sensors (Basel, Switzerland). 2017;17(6):1400.

29. Jiang $\mathrm{Y}, \mathrm{Pu} \mathrm{K}$. Advanced photoacoustic imaging applications of near-infrared absorbing organic nanoparticles. Small. 2017;13(30):1700710.

30. Lemaster JE, Jokerst JV. What is new in nanoparticle-based photoacoustic imaging? Wiley Interdiscip Rev Nanomed Nanobiotechnol. 2017;9(1):e1404.

31. Wang LV, Gao L. Photoacoustic microscopy and computed tomography: from bench to bedside. Annu Rev Biomed Eng. 2014;16:155-85. 
32. Menke J. Photoacoustic breast tomography prototypes with reported human applications. Eur Radiol. 2015;25(8):2205-13.

33. Xia J, Yao J, Wang LV. Photoacoustic tomography: principles and advances. Electromagnetic Waves (Cambridge, Mass). 2014;147:1-22.

34. Erpelding TN, Kim C, Pramanik M, Jankovic L, Maslov K, Guo $\mathrm{Z}$, et al. Sentinel lymph nodes in the rat: noninvasive photoacoustic and US imaging with a clinical US system. Radiology. 2010;256(1):102-10.

35. Bouchard LS, Anwar MS, Liu GL, Hann B, Xie ZH, Gray JW, et al. Picomolar sensitivity MRI and photoacoustic imaging of cobalt nanoparticles. Proc Natl Acad Sci USA. 2009;106(11):4085-9.

36. Jiao S, Xie Z, Zhang HF, Puliafito CA. Simultaneous multimodal imaging with integrated photoacoustic microscopy and optical coherence tomography. Opt Lett. 2009;34(19):2961-3.

37. Wang X, Chamberland DL, Carson PL, Fowlkes JB, Bude RO, Jamadar DA, et al. Imaging of joints with laser-based photoacoustic tomography: an animal study. Med Phys. 2006;33(8):2691-7.

38. Chamberland DL, Wang X, Roessler BJ. Photoacoustic tomography of carrageenan-induced arthritis in a rat model. J Biomed Opt. 2008;13(1):011005.

39. Wang X, Chamberland DL, Jamadar DA. Noninvasive photoacoustic tomography of human peripheral joints toward diagnosis of inflammatory arthritis. Opt Lett. 2007;32(20):3002-4.

40. Sun Y, Jiang H. Quantitative three-dimensional photoacoustic tomography of the finger joints: phantom studies in a spherical scanning geometry. Phys Med Biol. 2009;54(18):5457-67.

41. Sun Y, Sobel E, Jiang H. Quantitative three-dimensional photoacoustic tomography of the finger joints: an in vivo study. J Biomed Opt. 2009;14(6):064002.

42. Yuan Z, Jiang H. Three-dimensional finite-element-based photoacoustic tomography: reconstruction algorithm and simulations. Med Phys. 2007;34(2):538-46.

43. Sun Y, Sobel ES, Jiang H. First assessment of three-dimensional quantitative photoacoustic tomography for in vivo detection of osteoarthritis in the finger joints. Med Phys. 2011;38(7):4009-17.

44. van Es P, Biswas SK, Bernelot Moens HJ, Steenbergen W, Manohar S. Initial results of finger imaging using photoacoustic computed tomography. J Biomed Opt. 2014;19(6):060501.

45. Ermilov S, Zamora M, Oraevsky A. Optoacoustic angiography of peripheral vasculature. Proc Spie. 2012;8223(3):8.

46. Hagiwara Y, Izumi T, Yabe Y, Sato M, Sonofuchi K, Kanazawa $\mathrm{K}$, et al. Simultaneous evaluation of articular cartilage and subchondral bone from immobilized knee in rats by photoacoustic imaging system. J Orthopaed Sci. 2015;20(2):397-402.

47. Liu Y, Wang Y, Yuan Z. Dual-modality imaging of the human finger joint systems by using combined multispectral photoacoustic computed tomography and ultrasound computed tomography. Biomed Res Int. 2016;2016:1453272.

48. Oeri M, Bost W, Senegond N, Tretbar S, Fournelle M. Hybrid photoacoustic/ultrasound tomograph for real-time finger imaging. Ultrasound Med Biol. 2017;43(10):2200-12.

49. Guo H, Wang Q, Qi W, Sun X, Ke B, Xi L. Assessing the development and treatment of rheumatoid arthritis using multiparametric photoacoustic and ultrasound imaging. J Biophotonics. 2019;12(11):e201900127.

50. Wang X, Fowlkes JB, Cannata JM, Hu C, Carson PL. Photoacoustic imaging with a commercial ultrasound system and a custom probe. Ultrasound Med Biol. 2011;37(3):484-92.

51. Rajian JR, Girish G, Wang X. Photoacoustic tomography to identify inflammatory arthritis. J Biomed Opt. 2012;17(9):96013-21.

52. Rajian JR, Shao X, Chamberland DL, Wang X. Characterization and treatment monitoring of inflammatory arthritis by photoacoustic imaging: a study on adjuvant-induced arthritis rat model. Biomed Opt Express. 2013;4(6):900-8.

53. Xu G, Rajian JR, Girish G, Kaplan MJ, Fowlkes JB, Carson PL, et al. Photoacoustic and ultrasound dual-modality imaging of human peripheral joints. J Biomed Opt. 2013;18(1):10502.

54. Daoudi K, van den Berg PJ, Rabot O, Kohl A, Tisserand S, Brands $\mathrm{P}$, et al. Handheld probe integrating laser diode and ultrasound transducer array for ultrasound/photoacoustic dual modality imaging. Opt Express. 2014;22(21):26365-74.

55. van den Berg PJ, Daoudi K, Bernelot Moens HJ, Steenbergen W. Feasibility of photoacoustic/ultrasound imaging of synovitis in finger joints using a point-of-care system. Photoacoustics. 2017;8:8-14.

56. Jo J, Xu G, Cao M, Marquardt A, Francis S, Gandikota G, et al. A functional study of human inflammatory arthritis using photoacoustic imaging. Sci Rep. 2017;7(1):15026.

57. Zhu Y, Xu G, Yuan J, Jo J, Gandikota G, Demirci H, et al. Light emitting diodes based photoacoustic imaging and potential clinical applications. Sci Rep. 2018;8(1):9885.

58. Yang M, Zhao L, He X, Su N, Zhao C, Tang H, et al. Photoacoustic/ultrasound dual imaging of human thyroid cancers: an initial clinical study. Biomed Opt Express. 2017;8(7):3449-57.

59. Zhao C, Wang Q, Tao X, Wang M, Yu C, Liu S, et al. Multimodal photoacoustic/ultrasonic imaging system: a promising imaging method for the evaluation of disease activity in rheumatoid arthritis. Eur Radiol. 2021;31(5):3542-52. https://doi.org/10.1007/ s00330-020-07353-Z.

60. Xi L, Jiang H. Integrated photoacoustic and diffuse optical tomography system for imaging of human finger joints in vivo. J Biophotonics. 2016;9(3):213-7.

61. Ray A, Yoon HK, Koo Lee YE, Kopelman R, Wang X. Sonophoric nanoprobe aided $\mathrm{pH}$ measurement in vivo using photoacoustic spectroscopy. Analyst. 2013;138(11):3126-30.

62. Beziere N, von Schacky C, Kosanke Y, Kimm M, Nunes A, Licha $\mathrm{K}$, et al. Optoacoustic imaging and staging of inflammation in a murine model of arthritis. Arthritis Rheumatol (Hoboken, NJ). 2014;66(8):2071-8.

63. Chamberland DL, Agarwal A, Kotov N, Brian Fowlkes J, Carson PL, Wang X. Photoacoustic tomography of joints aided by an Etanercept-conjugated gold nanoparticle contrast agent-an ex vivo preliminary rat study. Nanotechnology. 2008;19(9):095101.

64. Keswani RK, Tian C, Peryea T, Girish G, Wang X, Rosania GR. Repositioning clofazimine as a macrophage-targeting photoacoustic contrast agent. Sci Rep. 2016;6:23528.

65. Zhao C, Zhang R, Luo Y, Liu S, Tang T, Yang F, et al. Multimodal VEGF-targeted contrast-enhanced ultrasound and photoacoustic imaging of rats with inflammatory arthritis: using dyeVEGF-antibody-loaded microbubbles. Ultrasound Med Biol. 2020;46(9):2400-11.

66. Ukai T, Sato M, Ishihara M, Yokoyama M, Takagaki T, Mitani $\mathrm{G}$, et al. Usefulness of using laser-induced photoacoustic measurement and 3.0 Tesla MRI to assess knee cartilage damage: a comparison study. Arthritis Res Therapy. 2015;17:383.

67. Biswas SK, van Es P, Steenbergen W, Manohar S. A method for delineation of bone surfaces in photoacoustic computed tomography of the finger. Ultrason Imaging. 2016;38(1):63-76.

68. Huang N, He M, Shi H, Zhao Y, Lu M, Zou X, et al. Curved-arraybased multispectral photoacoustic imaging of human finger joints. IEEE Trans Biomed Eng. 2018;65(7):1452-9. https://doi.org/10. 1109/TBME.2017.2758905.

Publisher's Note Springer Nature remains neutral with regard to jurisdictional claims in published maps and institutional affiliations. 